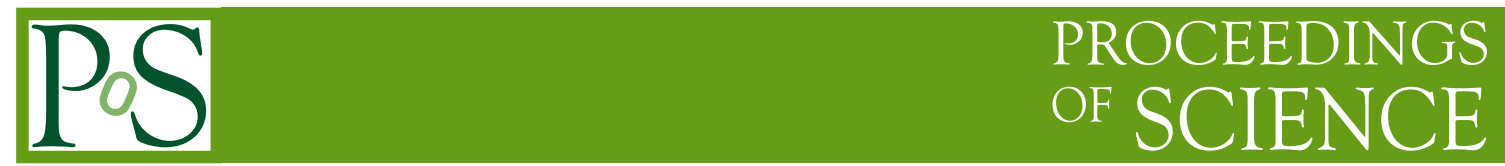

\title{
Improving bulk QCD thermodynamics on the lattice
}

\section{Edwin Laermann* with Frithjof Karsch ${ }^{\dagger}$ and Stanislav Shcheredin}

Fakultät für Physik, Universität Bielefeld, 33615 Bielefeld, Germany

E-mail: edwin@physik.uni-bielefeld.de

It is shown in the Stefan-Boltzmann limit that fermion actions with an improved dispersion relation lead to bulk thermodynamic quantities as the free energy which also have discretization errors at the same order of the lattice spacing only.

The XXV International Symposium on Lattice Field Theory

July 30 - August 42007

Regensburg, Germany

\footnotetext{
* Speaker.

$\dagger$ †also at Physics Department, Brookhaven National Laboratory, Upton, NY 11973, USA
} 


\section{Introduction}

Numerical QCD lattice computations of bulk thermodynamic quantities like energy density or pressure suffer from the fact that the signal decreases proportional to $1 / N_{\tau}^{4}$. One is therefore forced to carry out investigations into these quantities at fairly small values of the temporal lattice extent $N_{\tau}$. At fixed temperature $T \equiv 1 / a N_{\tau}$, this in turn amounts to relying on simulations on rather coarse lattices. It is therefore important to reduce the discretization effects by means of utilizing improved actions.

The use of discretization schemes which have been built to improve the Stefan-Boltzmann limit on the lattice has been observed to also lead to improvement in the interacting case [1]. Moreover, comparisons with alternative, non-lattice approaches as e.g. in [2] become more reliable if the high temperature limit is under control.

Here, we proof in this limit that fermion actions with a dispersion relation improved to $\mathscr{O}\left(a^{n}\right)$ also warrant bulk thermodynamic quantities to be affected by lattice artefacts only at the same order. We further give examples for fermions of the staggered as well as of Wilson type.

\section{The general case}

The dispersion relation $E(\vec{p})$ is obtained from the zeroes of the denominator $D$ of the quark propagator, e.g. in the naive discretization

$$
D\left(E=i p_{4}, \vec{p}\right)=0 \Leftrightarrow \sum_{k=1}^{3} \sin ^{2}\left(a p_{\mu}\right)-\sinh ^{2}(a E)=0
$$

In general, $D$ can be written as a polynomial in $\sin ^{2}\left(a p_{4}\right)$, (in $\sin ^{2}\left(a p_{4} / 2\right)$ for Wilson-type fermions),

$$
D\left(p_{4}, \vec{p}\right)=\sum_{i=0}^{p} A_{i}(\vec{p}) \sin ^{2 i}\left(a p_{4}\right)
$$

with coefficients $A_{i}(\vec{p})$. Rewriting $D$ in terms of its roots, $\omega_{i}(\vec{p})$,

$$
D\left(k_{4}, \vec{p}\right)=A_{p}(\vec{p}) \prod_{i=1}^{p}\left[\sin ^{2}\left(a k_{4}\right)+\omega_{i}^{2}(\vec{p})\right]
$$

(where $k_{4}=p_{4}$ for staggered and $k_{4}=p_{4} / 2$ for Wilson quarks) immediately gives the dispersion relation(s)

$$
\sinh ^{2}\left(a \varepsilon_{i}(\vec{p})\right)=-\sin ^{2}\left(a k_{4}\right)=\omega_{i}^{2}(\vec{p})
$$

(with $\varepsilon_{i}=E_{i}$ for staggered and $\varepsilon_{i}=E_{i} / 2$ for Wilson). Although $D$ is real, the roots can in general be complex. However, the root which survives the continuum limit, $\omega_{1}$ to be definite, is real.

For both, standard staggered and standard Wilson fermions the dispersion relation receives $\mathscr{O}\left(a^{2}\right)$ corrections,

$$
E^{2}(\vec{p})=p^{2}+\mathscr{O}\left(a^{2} p^{4}\right)+\mathscr{O}\left(a^{2} \sum_{k=1}^{3} p_{k}^{4}\right)
$$

with $p^{2}=\vec{p}^{2}=\sum_{k=1}^{3} p_{k}^{2}$. Improving the dispersion relation moves the leading $\mathscr{O}\left(a^{2}\right)$ lattice artefacts to some order $n$. For this purpose it suffices to construct an action with rotational invariance 
maintained to this order. To see how this works let us briefly discuss the dispersion relation for the standard staggered fermion action which is identical to Eq.(2.1). Expanding this relation up to terms of order $p_{\mu}^{4}, \mu=1,4$ yields

$$
E^{2}-p^{2}+\frac{1}{3} \sum_{k=1}^{3} a^{2} p_{k}^{4}+\frac{1}{3} a^{2} E^{4}=0
$$

If for an improved action the Euclidean propagator is rotationally invariant at $\mathscr{O}\left(p_{\mu}^{4}\right)$, i.e. terms $\sim \sum_{\mu=1}^{4} p_{\mu}^{4}$ are absent, the low momentum expansion of $D$ factorizes into

$$
\left(E_{1}^{2}-p^{2}\right)\left[1+\mathscr{O}\left(a^{2} p_{k}^{2}, a^{2} E_{1}^{2}\right)\right]+\mathscr{O}\left(a^{4} p_{\mu}^{6}\right)=0
$$

for the branch $E_{1}$ surviving the continuum limit such that the dispersion relation is $\mathscr{O}\left(a^{2}\right)$ improved.

Turning now to thermodynamics, the free energy of a free staggered fermion gas on the lattice is given [3] as (for Wilson fermions the sum extends up to $N_{\tau}-1$ )

$$
f \sim-\frac{N_{c}}{N_{\sigma}^{3} N_{\tau}} \sum_{\vec{p}} \sum_{j=0}^{N_{\tau} / 2-1} \ln \left[D\left(p_{4}, \vec{p}\right)\right]
$$

where $a p_{4}=\left(\pi / N_{\tau}\right)(2 j+1)$. In terms of the roots of the dispersion relation it can be rewritten as

$$
f \sim-\frac{8 N_{c}}{N_{\sigma}^{3} N_{\tau}} \sum_{\vec{p}} \sum_{j=0}^{N_{\tau} / 2-1}\left\{\ln A_{p}(\vec{p})+\sum_{i=1}^{p} \ln \left[\sin ^{2}\left(a p_{4}\right)+\omega_{i}^{2}(\vec{p})\right]\right\}
$$

with obvious generalization to Wilson quarks, see remark below Eq.(2.3). The important contribution in the continuum limit is due to the $i=1$ term in Eq.(2.9),

$$
\sum_{j=0}^{N_{\tau} / 2-1} \ln \left[\sin ^{2}\left(a p_{4}\right)+\omega_{1}^{2}(\vec{p})\right]=\ln \left[\frac{4}{2^{N_{\tau}}} \cosh ^{2}\left(\frac{N_{\tau} a E_{1}}{2}\right)\right]
$$

and the equality arises from carrying out the sum over $j$ [4]. Having subtracted the zero temperature result, the free energy is then obtained, for both staggered and Wilson type quarks, as

$$
f \sim-\frac{N_{c}}{N_{\sigma}^{3} N_{\tau}} \sum_{\vec{p}} 2 \ln \left[1+\exp \left(-N_{\tau} a E_{1}\right)\right]
$$

save terms which vanish exponentially in the continuum limit $N_{\tau} \sim 1 / a \rightarrow \infty$.

Suppose now that the dispersion relation is $\mathscr{O}\left(a^{n-2}\right)$ improved,

$$
E_{1}^{2}=p^{2}+\mathscr{O}\left(a^{n} p_{\mu}^{n+2}\right)
$$

In this case, the corrections to $E_{1} / T$ start at $\mathscr{O}\left(N_{\tau}^{-n}\right)$,

$$
\frac{E_{1}}{T}=N_{\tau} a E_{1}=\frac{p}{T}\left[1+\frac{1}{N_{\tau}^{n}}\left(\frac{p}{T}\right)^{n}\right]=\frac{p}{T}[1+\Delta]
$$

By expanding in $\Delta$ one finally arrives at

$$
f \sim-\frac{N_{c}}{N_{\sigma}^{3} N_{\tau}} \sum_{\vec{p}}\{\ln [1+\exp (-p / T)]-B \Delta\}
$$

where $B$ is given as $B=e^{-p / T} /\left(1+e^{-p / T}\right)$. Thus, improving the dispersion relation immediately leads to an improvement of the high temperature limit of free energy, pressure and energy density. 


\section{Staggered type quarks}

The Dirac matrix of a general class of staggered actions with fermion and anti-fermion fields separated by up to three links is given by

$$
\begin{aligned}
M(x, y)=\sum_{\mu} \eta_{\mu}(x)( & \sum_{i=1,3} c_{i, 0}[\delta(x+i \hat{\mu}, y)-\delta(x-i \hat{\mu}, y)] \\
& \left.+\sum_{v \neq \mu} \sum_{j= \pm 2} c_{1, j}[\delta(x+\hat{\mu}+j \hat{v}, y)-\delta(x-\hat{\mu}+j \hat{v}, y)]\right)
\end{aligned}
$$

Within this class one can construct actions that are rotationally invariant up to $\mathscr{O}\left(p^{4}\right)$. This can be achieved with the constraints [5]

$$
\begin{aligned}
& c_{1,0}+3 c_{3,0}+6 c_{1,2}=\frac{1}{2} \\
& c_{1,0}+27 c_{3,0}+6 c_{1,2}=24 c_{1,2}
\end{aligned}
$$

In particular we may set $c_{1,2} \equiv 0$. This yields the familiar Naik-action [6] with $c_{1,0}=9 / 16$ and $c_{3,0}=-1 / 48$. In this case the expansion of the dispersion to lowest orders gives

$$
E_{1}=p+\frac{3}{40}\left\{p^{5}-\frac{1}{p} \sum_{k} p_{k}^{6}\right\} a^{4}+\frac{1}{56}\left\{p^{7}+\frac{1}{p} \sum_{k} p_{k}^{8}\right\} a^{6}+\ldots
$$

Another choice is to eliminate the linear three link term completely, $c_{3,0} \equiv 0$ to obtain $c_{1,0}=3 / 8$ and $c_{1, \pm 2}=1 / 48$. Although this action, the $\mathrm{p} 4$ action [5] has $\mathscr{O}\left(a^{2}\right)$ corrections the $\mathscr{O}\left(\sum_{k} p_{k}^{4}\right)$ terms are eliminated from the propagator by construction,

$$
a^{-2} D\left(E_{1}, \vec{p}\right)=\left(E_{1}^{2}-p^{2}\right)\left[1+\frac{a^{2}}{3}\left(E_{1}^{2}+p^{2}\right)\right]+\mathscr{O}\left(a^{4}\right)
$$

such that the dispersion relation is $\mathscr{O}\left(a^{2}\right)$ improved, with the expansion

$E_{1}=p+\frac{3}{40}\left\{p^{5}-\frac{1}{p} \sum_{k} p_{k}^{6}\right\} a^{4}+\frac{1}{4536}\left\{25 p^{7}-112 p \sum_{k} p_{k}^{6}-84 \frac{1}{p}\left(\sum_{k} p_{k}^{4}\right)^{2}+165 \frac{1}{p} \sum_{k} p_{k}^{8}\right\} a^{6}+\ldots$

Note that the leading correction is the same as for the Naik action, the higher orders are generally smaller. In the comparison of the dispersion relations for standard, Naik and $\mathrm{p} 4$ action, shown in Figure 1 (left), we have chosen momenta along the z-direction.

Following the dispersion relations, the deviations from the continuum of the free energies start at $\mathscr{O}\left(N_{\tau}^{-4}\right)$ for both, Naik and $\mathrm{p} 4$ action,

$$
\begin{aligned}
f^{\text {Naik }} / f_{S B} & =1-\frac{1143}{980}\left(\frac{\pi}{N_{\tau}}\right)^{4}-\frac{365}{77}\left(\frac{\pi}{N_{\tau}}\right)^{6}+\ldots \\
f^{\mathrm{p} 4} / f_{S B} & =1-\frac{1143}{980}\left(\frac{\pi}{N_{\tau}}\right)^{4}+\frac{73}{2079}\left(\frac{\pi}{N_{\tau}}\right)^{6}+\ldots
\end{aligned}
$$



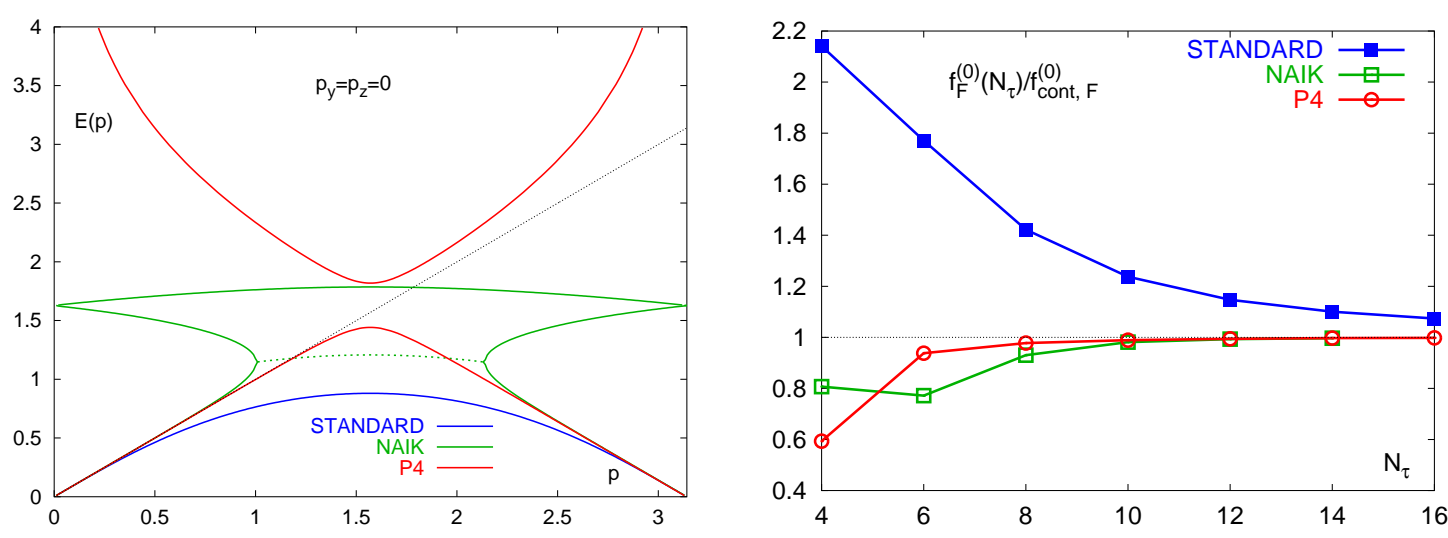

Figure 1: Dispersion relations (left) and free energies (right) for standard staggered quarks, the Naik and the p4 action. For the dispersion relations, a momentum along the z-direction has been chosen.

The leading corrections are of course the same for both improved actions, yet, the next to leading one is considerably smaller in the 44 case. This is also reflected in the complete free energies which are depicted in Figure 1 (right). Due to their improved dispersion relations, the free energies for Naik and p4 are close to the continuum Stefan-Boltzmann limit already at small temporal lattice extents, in particular, for $\mathrm{p} 4$ the deviations from continuum at $N_{\tau}=6$ are merely a few per cent.

\section{Wilson type quarks}

The Dirac matrix of a generic Wilson type action with couplings constrained to a hypercube of size $3^{4}$ can be written as

$$
M(x, y)=\sum_{\mu=1}^{4} \gamma_{\mu} \rho_{\mu}(x-y)+\lambda(x-y)
$$

with

$$
\begin{aligned}
\rho_{\mu}(x-y)= & \rho_{1}[\delta(y, x+\hat{\mu})-\delta(y, x-\hat{\mu})]+\rho_{2} \sum_{\hat{v}}[\delta(y, x+\hat{\mu}+\hat{v})-\delta(y, x-\hat{\mu}+\hat{v})] \\
& +\rho_{3} \sum_{\hat{v}, \hat{\rho}}[\delta(y, x+\hat{\mu}+\hat{v}+\hat{\rho})-\delta(y, x-\hat{\mu}+\hat{v}+\hat{\rho})] \\
& +\rho_{4} \sum_{\hat{v}, \hat{\rho}, \hat{\sigma}}[\delta(y, x+\hat{\mu}+\hat{v}+\hat{\rho}+\hat{\sigma})-\delta(y, x-\hat{\mu}+\hat{v}+\hat{\rho}+\hat{\sigma})]
\end{aligned}
$$

for the vector terms and

$$
\begin{aligned}
\lambda(x-y)= & \lambda_{0} \delta(y, x)+\lambda_{1} \sum_{\hat{\mu}}[\delta(y, x+\hat{\mu})+\delta(y, x-\hat{\mu})]+\lambda_{2} \sum_{\hat{\mu}, \hat{v}}[\delta(y, x+\hat{\mu}+\hat{v})+\delta(y, x-\hat{\mu}+\hat{v})] \\
& +\lambda_{3} \sum_{\hat{\mu}, \hat{v}, \hat{\rho}}[\delta(y, x+\hat{\mu}+\hat{v}+\hat{\rho})+\delta(y, x-\hat{\mu}+\hat{v}+\hat{\rho})] \\
& +\lambda_{4} \sum_{\hat{\mu}, \hat{v}, \hat{\rho}, \hat{\sigma}}[\delta(y, x+\hat{\mu}+\hat{v}+\hat{\rho}+\hat{\sigma})+\delta(y, x-\hat{\mu}+\hat{v}+\hat{\rho}+\hat{\sigma})]
\end{aligned}
$$


for the scalar ones. The sums over $\hat{v}, \hat{\rho}, \hat{\sigma}$ extend over positive and negative directions and are mutually orthogonal to each other and to $\hat{\mu}$. The general action is subject to the contraints

$$
\begin{aligned}
2 \rho_{1}+12 \rho_{2}+24 \rho_{3}+16 \rho_{4} & =1 \\
\lambda_{0}+8 \lambda_{1}+24 \lambda_{2}+32 \lambda_{3}+16 \lambda_{4} & =0
\end{aligned}
$$

to reproduce the continuum dispersion relation for $a \rightarrow 0$, see also [7]. Examples for this type of action are the standard Wilson action (including the clover improved version of it) and the hypercube truncated perfect action [8,9], with coefficients as listed in Table 1 for the massless case.

\begin{tabular}{|l|r|c||c|r|c|}
\hline & Hypercube & Wilson & & Hypercube & Wilson \\
\hline \hline & & & $\lambda_{0}$ & 1.852720547 & 4 \\
$\rho_{1}$ & 0.136846794 & $1 / 2$ & $\lambda_{1}$ & -0.060757866 & $-1 / 2$ \\
$\rho_{2}$ & 0.032077284 & 0 & $\lambda_{2}$ & -0.030036032 & 0 \\
$\rho_{3}$ & 0.011058131 & 0 & $\lambda_{3}$ & -0.015967620 & 0 \\
$\rho_{4}$ & 0.004748991 & 0 & $\lambda_{4}$ & -0.008426812 & 0 \\
\hline
\end{tabular}

Table 1: Coefficients $\rho_{i}$ and $\lambda_{i}$ for standard Wilson quarks and for the hypercube action.

The resulting dispersion relations are shown in Figure 2 (left), together with the leading correction to the continuum

$$
E_{1}=p-\frac{\rho_{1}-12 \rho_{3}-16 \rho_{4}}{3}\left(p^{3}+\frac{1}{p} \sum_{k=1}^{3} p_{k}^{4}\right) a^{2}+\ldots
$$

where the first constraint from Eq.(4.4) has been exploited. Note that the scalar coefficients $\lambda_{i}$ do not appear in Eq.(4.5). It is worth mentioning that the corrections start at $\mathscr{O}\left(a^{2}\right)$ also in the standard Wilson case where the action deviates from the continuum at order $a$. However, the combination of couplings constituting the coefficient of the $a^{2}$-term amounts to +0.167 for standard Wilson but at -0.024 is considerably smaller for hypercube fermions. Small adjustments of the vector coefficients $\rho_{i}$ may easily allow to eliminate these $\mathscr{O}\left(a^{2}\right)$ corrections altogether.

Corresponding to the dispersion relation, Eq.(4.5), the leading correction to the continuum free energy starts at $\mathscr{O}\left(N_{\tau}^{-2}\right)$,

$$
f^{\mathrm{W}-\mathrm{type}} / f_{S B}=1+\frac{496}{147}\left(\rho_{1}-12 \rho_{3}-16 \rho_{4}\right)\left(\frac{\pi}{N_{\tau}}\right)^{2}+\ldots
$$

This correction is shown in Figure 2 (right), together with the complete result. While the curves for the $\mathscr{O}\left(N_{\tau}^{-2}\right)$ correction reflect the opposite sign of the coefficient and the difference in its value of about a factor 7 between Wilson and hypercube fermions, it is interesting to note that in the Wilson case the contributions from the subleading terms are large at small $N_{\tau}$ and only slowly vanish with rising temporal extent whereas they are negligeable for hypercube fermions.

\section{Conclusion}

In the Stefan-Boltzmann limit we have generally shown and subsequently exemplified for various staggered and Wilson type fermion discretization schemes that improving the fermion dispersion relation to some order $a^{n}$ leads to bulk thermodynamic quantities improved to the same 

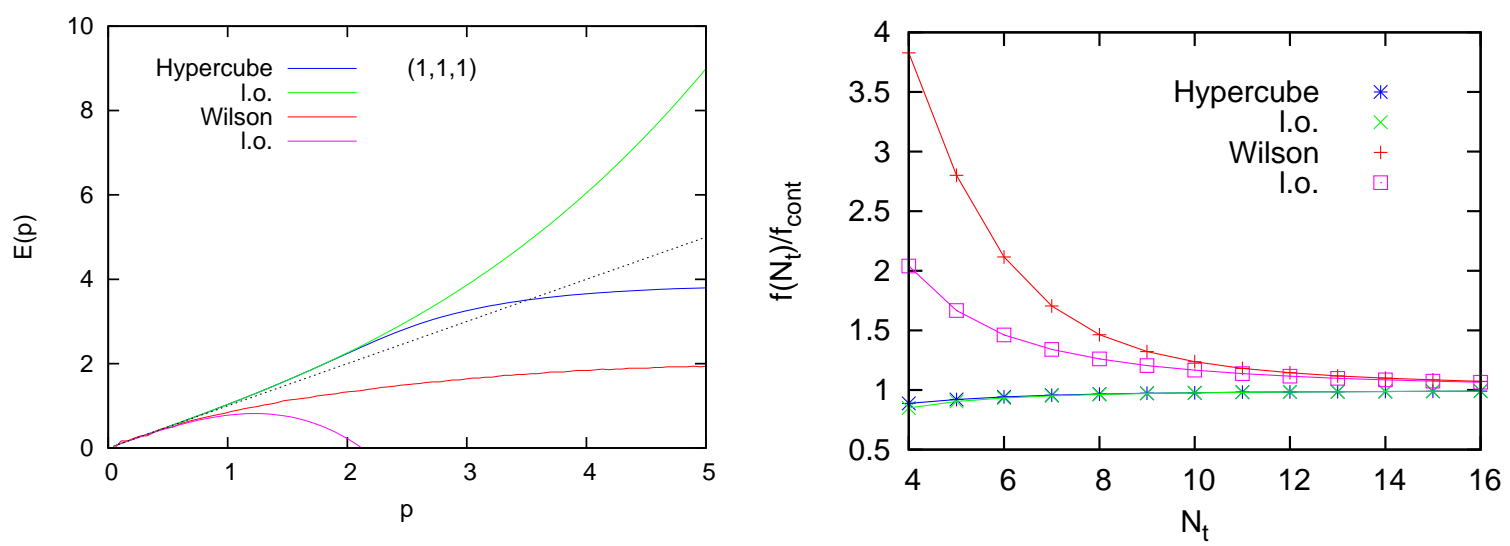

Figure 2: Dispersion relations (left) and free energies (right) for standard Wilson quarks and the hypercube truncated fixed point action. For the dispersion relations a momentum axis of $(1,1,1)$ has been chosen. Denoted by 1.o. the figure also shows the sum of continuum plus leading $\mathscr{O}\left(a^{2}\right)$ correction to the continuum behavior. The full results have also been shown in [9]

order in the lattice spacing. This discussion can be carried over to so-called chiral actions and to the case of non-vanishing chemical potential [10] and may provide some guidance in constructions of improved actions.

\section{Acknowledgments}

SS has been supported by the EU under contract no. RII3-CT-2004-506078. FK and EL acknowledge partial support through the BMBF grant 06BI106. The work of FK has been supported by a contract DE-AC02-98CH10886 with the U.S. Department of Energy.

\section{References}

[1] B. Beinlich et al., Eur. Phys. J. C6 (1999) 133.

[2] K. Kajantie et al., Phys. Rev. D67 (2003) 105008; F. Di Renzo et al., JHEP 0607 (2006) 26.

[3] J. Engels et al., Nucl. Phys. B205 (1982) 239.

[4] H.-Th. Elze, K. Kajantie and J. Kapusta, Nucl. Phys. B304 (1988) 832.

[5] F. Karsch, U. Heller and B. Sturm, Phys. Rev. D60 (1999) 114502.

[6] S. Naik, Nucl. Phys. B316 (1989) 238.

[7] D.H. Adams, Nucl. Phys. (Proc. Suppl.) 129\&130 (2004) 513.

[8] W. Bietenholz and U.-J. Wiese, Nucl. Phys. B464 (1996) 319.

[9] W. Bietenholz et al., Nucl. Phys. (Proc. Suppl.) 53 (1997) 921.

[10] P. Hegde et al., work in progress. 